\title{
Enabling Self-Reflection with LifelogExplorer: Generating Simple Views from Complex Data
}

\author{
Rafal Kocielnik \\ Eindhoven University of Technology \\ The Netherlands \\ Email: r.d.kocielnik@tue.nl
}

\author{
Fabrizio Maria Maggi \\ University of Tartu \\ Estonia \\ Email: f.m.maggi@ut.ee
}

\author{
Natalia Sidorova \\ Eindhoven University of Technology \\ The Netherlands \\ Email: n.sidorova@tue.nl
}

\begin{abstract}
Nowadays, people are overwhelmed with multiple tasks and responsibilities, resulting in increasing stress level. At the same time, it becomes harder to find time for self-reflection and diagnostics of problems that can be source of stress. In this paper, we propose a tool that supports a person in selfreflection by providing views on life events in their relation to person's well-being in a concise and intuitive form. The tool, called LifelogExplorer, takes sensor data (like skin conductance and accelerometer measurements) and data obtained from digital sources (like personal calendars) as input and generates views on this data which are comprehensible and meaningful for the user due to filtering and aggregation options which help to cope with the data explosion. We evaluate our approach on the data collected from two case studies focused on addressing stress at work: 1) with academic staff of a university, and 2) with teachers from a vocational school.
\end{abstract}

\section{INTRODUCTION}

In today's society, people are overwhelmed with multiple tasks and responsibilities, often resulting in disturbing stress balance. The growing "digital world" and abundance of information poses the challenge to our ability to actively and consciously process, understand and cope with the everyday demands by ourselves [1]. The increasing popularity of relaxation and reflection techniques, like meditation, yoga, personal coaching, is evidence of a widespread need in contending with stress [2], [3]. At the same time, due to this overwhelming demand, it is often hard to find time for (self)reflection and diagnostics of problems that can be source of stress and the ways to address them efficiently. Furthermore, the large amount of tasks we perform makes it difficult to connect the reactions of our body with the direct and indirect triggers of those reactions. It is important to motivate people to adjust their behavior and life style and to use appropriate stress coping strategies far before the increased level of stress results in serious health problems. Therefore we opt for early signaling and treatment of stress problems by introducing inexpensive, unobtrusive, and widely available technologies for creating awareness of the objective level of stress and understanding the specific contexts in which it is experienced.

In this paper, we propose a tool for facilitating selfreflection and identification of stress-related issues by visualizing links between stress-related data and data reflecting other aspects of person's life. There is a lot of information about person's activities that can be extracted from the digital world. Indeed, in many of our everyday activities, we use systems producing a large amount of digital objects, like digital calendars, e-mails, pictures, social-network messages, (logs of) phone calls. Physical activities and physiological responses of the body can now be captured and digitalized easier than before, thanks to developments in wearable sensor technologies [4], making an automated capture and storage of everyday events, also called lifelogging, feasible.

In this paper, we describe the LifelogExplorer, a tool whose main purpose is to enable self-reflection. It presents to the user concise and intuitive views on data about his/her life obtained from multiple sources in a way that is meaningful and helpful for him/her. To achieve that, we (1) build upon the platform described in [5] to capture and process lifelog data, including sensor data and digital world data in an automated way, (2) interpret and align data from multiple information sources and generate views on the aligned data with our visualization tool to provide the user with (self-)reflection material in a concise and intuitive form, and (3) evaluate our approach on two case studies: one performed with university staff and another with teachers of a vocational school, both focused on the issue of work-related stress.

Since the life $\log$ is inherently incomplete, the user's knowledge about his/her life is essential for drawing conclusions. Our LifelogExplorer aims rather at presenting the user with the views on the user's past, allowing the user to discover patterns in his/her reactions to different things. Since we generate views on data for several weeks of monitoring, including dozens of events, LifelogExplorer offers options for grouping and filtering information and summarizing data across different dimensions, thus allowing to avoid information explosion. Due to this options, the user is free to choose what is interesting and relevant for him, which facilitates the capture of personally meaningful trends. Our solution is not limited to the time dimension but also offers a set of meaningful cues based on meta-information connected with events, like people involved, locations and conversation topics.

In our case studies, we collected sensor and calendar data of study participants in their normal working conditions for the period of at least 7 weeks per participant, generated views on their data with LifelogExplorer and evaluated them in semistructured interviews with the participants. We present the results of these evaluation, confirming that LifelogExplorer supports self-reflection and triggers self-coaching for achieving a better stress balance.

The rest of this paper is organized as follows. In the Section II, we describe the domain context and closely related works. In the Section III, we describe how we generate the 
LifelogExplorer views from various information sources in a meaningful way. In Section IV, we demonstrate the functionality of the LifelogExplorer on data sets obtained in two case studies we carried out and describe the results obtained in the evaluation with the users. In the Section V, we summarize our approach, its benefits and future steps.

\section{DOMAIN CONTEXT AND RELATED WORK}

The approach we took when designing the LifelogExplorer touches on many fields. In these section, we briefly discuss the links with them.

\section{A. Stress detection}

In our case studies, we primarily focus on the problem of stress at work. There are a lot of studies indicating the increase in the stress-related problems, especially in the work context. In the 2000 European Working Conditions Survey (EWCS) [6], work-related stress was found to be the second most common work-related health problem across the EU. Stress can contribute to illness directly, through its physiological effects or indirectly, through maladaptive health behaviors (for example, smoking, poor eating habits or lack of sleep) [7]. Yet, the avoidance of stress in the everyday working environment is impossible.

From the technical point of view, various emotional states of a person are reflected in physiological responses that can be objectively measured using various sensor technologies. Sensor data about skin conductance (also known as galvanic skin response) and heart rate variability, supplemented with information about physical activities (derivable from sensor data), can be used to estimate the stress level of the user [8]. Until recently, most of the approaches were used and tested in artificial lab conditions, where good recognition accuracies were reported. Recent advances in wearable sensor technologies enabled stress detection in everyday environments [9].

\section{B. Approaches enabling self-reflection and self-diagnostics}

Despite the recent upsurge of interest, the systems performing various forms of lifelogging are not new. Early digital implementations [10], [11] focused on storing collections of heterogeneous digital objects that users generate or use, like digital documents, photos, web pages, and e-mails. Lifelogging systems allow users to structure and categorize the information by providing facilities for searching, browsing, filtering and retrieving relevant information from the collections. However, lifelogging projects make more emphasis on the production of lifelogs with an extensive use of existing digital technologies than on the way the information is used to support specific goals. Tightly related to the lifelogging field, are various Quantified-Self approaches [12], focused on collecting longterm detailed data about a person to support making optimal health and behavioral choices in life. Members of the Quantified-Self community can be described as early adopters willing to use sometimes highly obtrusive technologies that would be not acceptable for other people.

The field of personal informatics addresses a class of systems that help people collect personally relevant information for the purpose of self-reflection and gaining selfknowledge [13], emphasizing two aspects: 1) these systems help people better understand their behavior, 2) people participate in the collection, exploration and understanding of the information. Furthermore, the authors define a model of personal informatics systems based on the stages of preparation, integration, reflection and action.

\section{Stress analytic}

In our approach, we build upon the framework we described in [5], where we proposed to analyse stress, estimated based on data from wearable sensors, in the context of the user's calendar events. Another recent work by [14] describes AffectAura-a system aimed at enabling self-reflection on emotional states over an extended period of time (one week in their use cases). The authors use an extensive setup for sensor measurements consisting of multiple sensors: Webcam, Kinect, Microphone EDA, GPS, File Activity, Calendar, in order to capture the precise context of emotional responses. AffectAura shows the emotional states on a linear time scale as "blobs" characterized by their color, shape and opacity, the color being linked to valence, the shape to arousal, and the opacity to engagement. Additionally, it provides context cues linked to each of the presented "blobs". A user study confirms that the users were able to use the provided context cues to construct stories about their day, even after they had forgotten some incidents or their related emotional tones. The results obtained in this work further confirm the practical need and usefulness of such systems. In our view, two aspects of this approach should be further elaborated on: Firstly, the extensive sensor setup limits the applicability of the approach to office-workers, missing other important groups, as the authors point out themselves. Secondly, the visualization of the data representation is driven by the time dimension only, limiting the possibility of discovering patterns related to other dimensions, like subjects of meetings, people involved, etc.

Unlike [5] and [14], the AffectiveHealth (www.sics.se/ah) project focuses on live presentation of the physiological signals with the main focus on providing real-time, short-term feedback to the user. The measurements are visualized in the form of a dynamic spiral to encourage personal reflection on body reactions throughout the day [15]. The recall and the pattern discovery are out of the scope in that approach.

In the Feel project, the authors propose a system for automated annotation and monitoring of phone calls and stress responses of the users [16] using a mobile phone and a wearable biosensor. They perform a real-time analysis for stress recognition and associate the recognized stress levels to the events connected with the use of the mobile phone. The event data and the associated stress levels are placed in a searchable journal to facilitate user reflection on his/her physiological responses. The visualization of the data is restricted to calendar views: day, week, month and a list view for events, limiting the ability of the user to spot non-time-related trends.

Activities of an individual, like walking, writing, etc. can provide additional context; these cues can be measured either by wearable sensors [17] or by user-free sensor networks placed in the environment and on the objects the user interacts with. Even using a single accelerometer, it is possible to recognize specific activities, as shown in [17], [18]. 


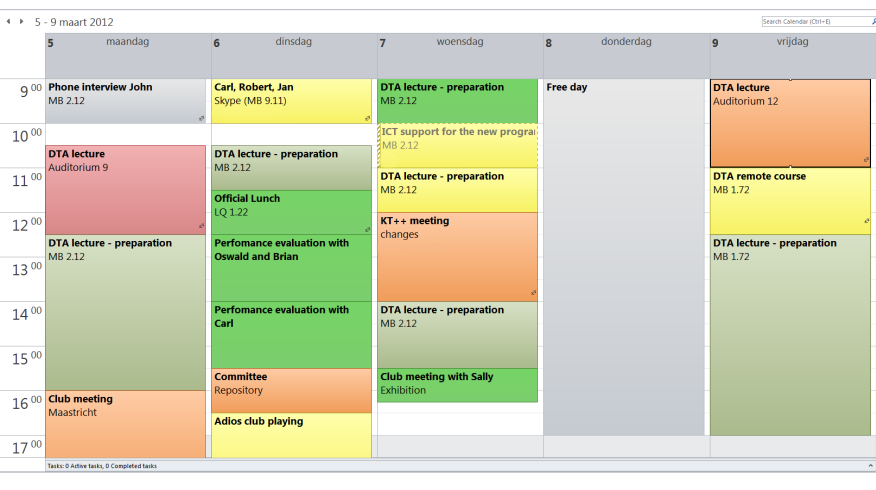

Fig. 1. A calendar view reinforced with information about arousal.

\section{APPROACH}

The main purpose of our tool - LifelogExplorer - is to enable self-reflection of the user by providing comprehensible and concise views on the lifelog data, including data stored in digital calendars and sensor data. These views summarize data collected for a particular user and allow the user to explore correlations between his/her reactions to the things that happened during the monitoring period with different levels of granularity. We do not assume the lifelog to be complete to such an extent to reveal the stress sources without relying on the user's knowledge, we only aim at providing help in recollecting the past. Then, the user is to draw conclusions about the stress sources and to find patterns in his/her behavior.

\section{A. Our information sources}

In our approach, we combine a number of distinct data sources, which have their own characteristics and are traditionally visualized in a number of different ways. In the following paragraphs we describe the data types we collect in our case studies, briefly discuss standard methods of visualizing such data and the advantages and disadvantages of these visualization types with respect to enabling recollection and self-reflection.

Digital calendars like the Outlook calendar are a widely used way to reinforce one's memory in daily life situations, mostly used to remember which events are to come in the future. The calendar data can also be used to take a look at the past. Calendar data can be seen as a time series of events with information about what, where, with whom, when and on whose initiative happened in the past.

The calendar view can be extended by supplementing it with information obtained from sensors (e.g., arousal information), like in the approach described in in [5]. An example of standard calendar view reinforced with information about arousal is shown in Fig. 1. The arousal scale ranges from green (very low arousal) to red (very high arousal), while grey indicated the absence of arousal information for an event.

Calendar tools have clear benefits for understandability. However, since their primary goal is to support person's memory about the events to come, the visualization of a calendar (as provided by standard tools) is not meant to support filtering and aggregation of data. It is also not meant to support long term interpretation of the events in connection with reactions of

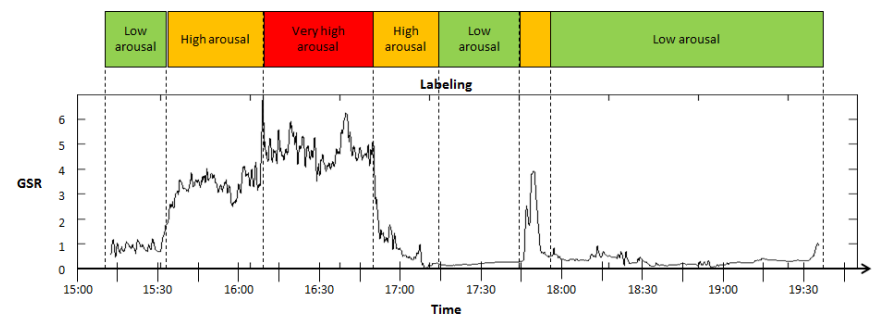

Fig. 2. Visualizing skin conductance in its raw form with associated labels.

the body. Even if directly augmented by data from sensors, still the calendar based views only offer a set of views at different time granularity, like day, week, month, year. In this case, no easy aggregation by other attributes, like subjects, locations, attendees, etc. is feasible. In conclusion, the calendar view, although very intuitive and familiar to the users, does not allow for expression of non-time based relations between events.

The skin conductance signal is a one dimensional timeseries data representing measured activity of sweat glands, which is often used (also in combination with other signals) for estimating the stress level of the user [19]. The standard way of visualizing the timeseries data is to plot the raw curve on a line graph. It is possible to filter out the noise from the signal, smooth it and provide interpretations by assigning labels to time periods resulting in a view like the one in (Fig. 2). Such a visualization is mostly useful in experimental conditions, when the time is limited and specific, and precise onsets of events are known. The raw signal is in this case inspected for expected emotional responses. The main drawbacks of this form of visualization are: 1) it is only directly meaningful to the expert that is able to provide some interpretation, 2) the ability to compare different time periods is limited, because plotting several curves together or overlapping them makes the plot difficult to read, 3) the visualization of signal for long time periods is incomprehensible, 4) it does not provide support for recollection, since the arousal information is presented without any context information (subjects, locations, etc.).

Another time series data that is useful in our approach is data reflecting everyday activities of an individual, like walking, writing, typing, etc. This data can come from different sources such as accelerometers. An example of raw data produced by an accelerometer can be seen in Fig. 3. As in case of other time series data, the same drawbacks to the visualization apply, with additional two in this case: 1) multiple channels make data even less clear to interpret than the one dimensional data, 2) the interpretation of the raw signal can be made at different, overlapping levels.Other types of sensor data captured, like ambient and skin temperature, ambient lighting, have similar form of the raw signal and therefore suffer from the same visualization issues.

\section{B. LifelogExplorer}

In the LifelogExplorer, we want to provide the user with information about the past from different points of view. It may be a time-related view, e.g., showing weekly patterns or views focusing on some not time-related data, e.g., subjects of calendar events or type of activities done such as writing, speaking, working at a computer. 


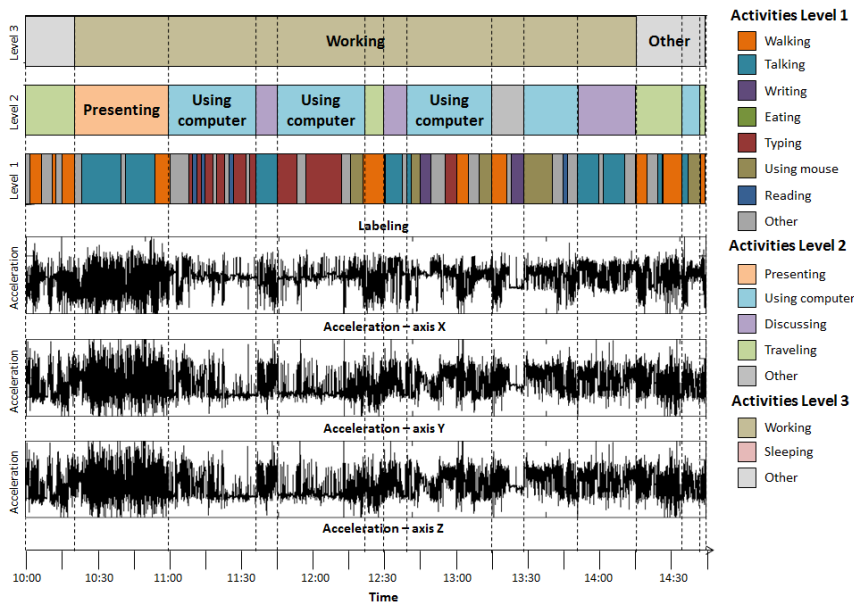

Fig. 3. 3D acceleration data in the raw form aligned with associated multilevel activity type labels.

We interpret the data obtained from different sources to construct a sequence of user's "states". Each state has several attributes such as time it was entered and left, activities the user was performing in this state, arousal level, body temperature, posture, environment temperature. In the interpretation of data, we often move to more abstract values for a number of parameters remaining stable for a longer period of time (e.g., it does not make sense to capture fluctuations of the environment temperature by $0.1^{\circ} \mathrm{C}$ ). The interpretations can replace pieces of signal with meaningful labels. The labeling can be performed on different abstraction levels: on one level, the labels could indicate that a person was writing for 10 minutes, then talking for 5 minutes, followed by listening to someone for 15 minutes. All these activities took place during a meeting with a group of people at work according to the calendar data. On another level this period might have been labeled as a discussion of 30 minutes. These interpretation are equally justifiable, although they interpret the data differently. The procedure used for obtaining arousal labels from sensor measurements is presented in [20].

The state representation we choose makes it possible to create an interactive visualization for aggregating, filtering and exploring correlations in the data from multiple perspectives. The large number of dimensions in the data space (reflected in the number of state attributes) makes it unpractical and infeasible to put all of them in one view. On the other hand, improper removal of some pieces of the data can suggest misleading interpretations. Ideally, one would like to see the full data from different perspectives to capture relevant aspects based on the goal at hand. Our solution to this problem is to provide aggregation of information on multiple levels.

Also, we do not aim at detecting stress predictors by using, for example, data mining techniques. In this way, we would only detect strong correlations, not causalities, and showing them to a user with a weak (or no) background in statistics might easily lead to misinterpretations. For example, the user could unconsciously draw wrong implications about stress sources ("whenever I meet John, I have a high arousal, so he is a serious source of my stress", while the correct reasoning might be "I always have long days on Thursdays, and Friday mornings always start with meetings with John, so no wonder that I have a higher arousal during these meetings, so early in the day, after having too little sleep, and my work relationship with John suffers from that").

We let the user to choose the "aggregation dimension" (e.g., days of the week) which triggers the partitioning of states according to the categories of the chosen data dimension. Every category is represented by a circle in the view we generate (a circle will represent, e.g., a day of the week or a subject used in the calendar or a location). Each circle represents thus a collection of states that belong to the same category, e.g., share the same value of attribute X. The size of a circular node represents the total time the user spent in the states aggregated in that circle.

To characterize a collection $C$ of the states represented by a circle $c$ from some other "characterization dimension", we associate a pie chart to every circle. The characterization dimension chosen defines the partitioning of each collection $C$ into subcollections $S \subseteq C$ of states, with a slice $s$ of the pie chart representing a subcollection. For example, the user can choose the arousal perspective, with a slice representing the states of this day of the week with arousal values, categorized in the slices into "very low", "low", "medium", "high" and "very high". The number of slices is defined by the number of categories. The size of a slice $s$ represents the total time spent in the states of the corresponding subcollection $S$ in relation to the time spent in the states of $C$. Examples of other perspectives are user activities (like writing, talking, typing, writing), locations, number of emails sent, etc.

The prevention of data clutter by aggregation might be not enough. In some situations it is useful to keep the aggregation at a low level, but be able to pick the data that is useful for the user's purpose. Therefore, we provide filtering to narrow the view to the things which are meaningful and relevant for the user. We obtain the filtering labels from the meta-data and let the user to select filters. Examples of such filters are subjects (e.g., only lectures), attendees (e.g., only John and Marc), locations (e.g., only at the university).

To extend our view with an additional dimension, we choose for a bipartite graph as a representation form: boxes or human-shaped nodes can be used for the "secondary dimension". For the sake of readability, we refer to these nodes as boxes. The boxes are associated with the circles by arrows, with an arrow from a box $b$ to a circle $c$ showing that there is a state in the collection $C$ represented by the circle $c$ that falls into the category represented by the box $b$ (because of its attribute values). In case there are too many categories for the secondary dimension, implying too many boxes and arrows, we allow for grouping the boxes linked to the same set of circles into a "crowd", showing the names of the crowd elements when the mouse points to it in the view. If the grouping into crowds option is not chosen, the elements belonging to the same crowd are depicted separately, but in their "crowd color".

The example in Fig. 4, generated on a data set from our case study (renaming was applied for privacy reasons), shows the weekly arousal view with the information about the weekly attendees patterns. Circles represent the state collections for the days of the week, with pie charts colored using the partitioning on arousal. As specified in the legend, colors range from green 


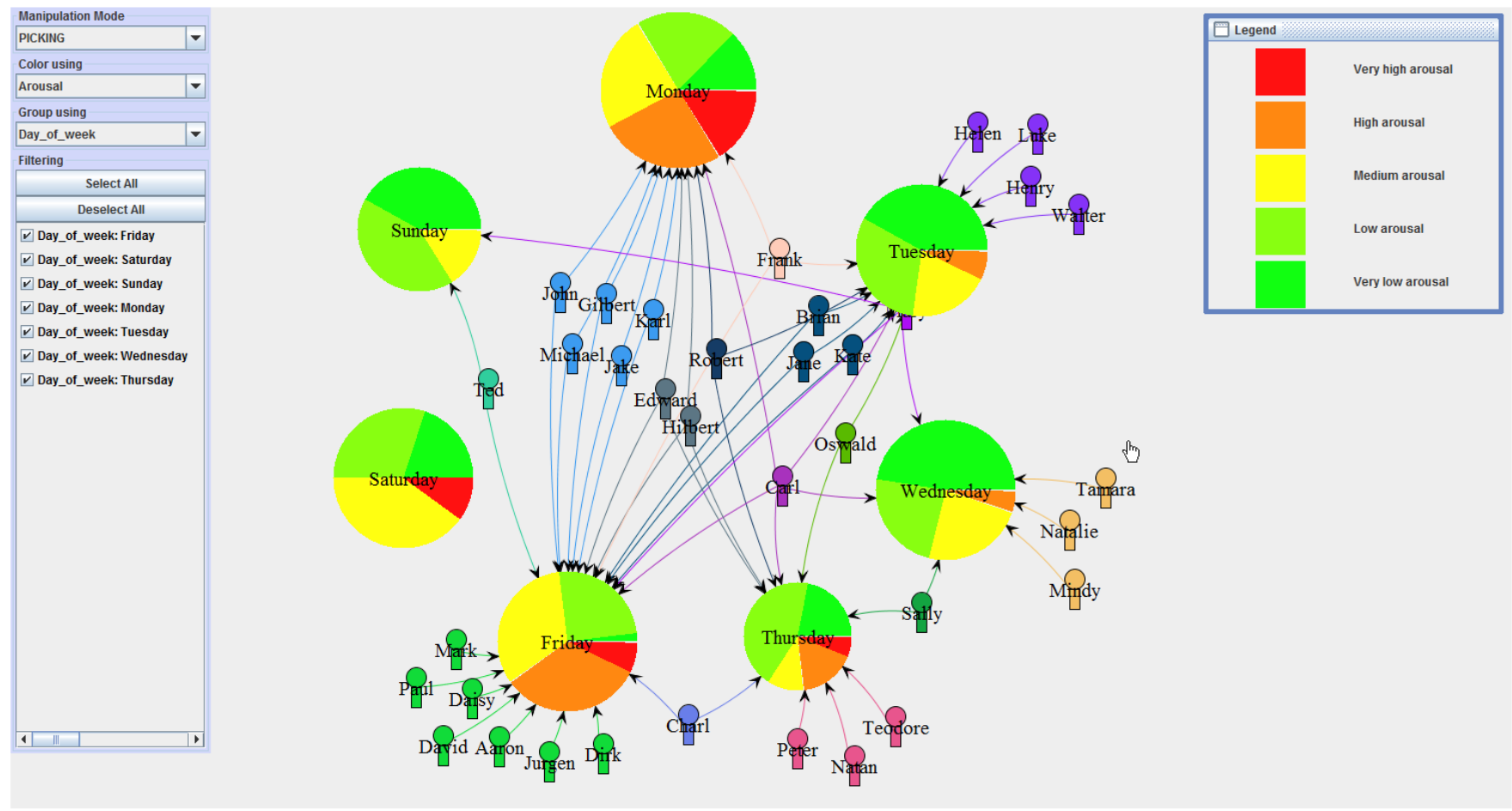

Fig. 4. A LifelogExplorer view where circles represent the days of the week and human-shaped nodes represent people present at the meetings on these days; colors represent measured arousal level.

to red, representing arousal values ranging from very low to very high. So Mondays and Fridays are days with the highest arousal, while Sundays are the most quiet compared to the other days of the week. Boxes (in this case human-shaped forms) represent the attendees of the meetings in the user's calendar. For example, the user met both Gilbert and Jake on a Monday and on a Friday.

\section{CASE STUDIES}

We evaluated our approach in two case studies: 1) with nine university staff members, 2) with five teachers of a vocational school. We used the Discrete Tension Indicator (DTI-2), a wrist-worn sensor device developed by Philips Research [9] that measures skin conductivity, 3D acceleration, band temperature (which is an estimation of the ambient temperature), skin temperature, and ambient illumination. The device stores recorded sensor data on an internal SD card, or streams it using Bluetooth.

The participants wore DTI-2 every day for at least seven weeks mainly during working hours (they were allowed to use it outside of these hours if they wanted to). In addition to the sensor measurements, we also collected their calendar information. At the end of each day, the participants were uploading the collected data (measurement + calendar) remotely to our server using a custom-made calendar application, which supports synchronization with Outlook and extends the data about calendar events with the subjective data about the emotional stress as perceived by the user; the subjective stress information about all events of a day was entered by the users at the end of that day. The users were given no feedback about the interpretation of the data during this period. In the following paragraphs we briefly summarize the collected data, the evaluation and its results for each of the studies.

\section{A. Study with university employees}

The participants were not supervised directly and the interventions were made on participants' initiative only. This resulted in 37 days with data per user, on average. There were 188 calendar entries per participant on average, 110 of which, on average, took place when a participant was also wearing the DTI-2 device, which amounts to $44 \%$ of coverage of calendar activities by sensor measurements.

Evaluation procedure: The evaluation was in the form of a semi-structured interview in which we focused on qualitative results, in which we used six views with the same dimensions for every participant, where attendees were used for the secondary dimension in the view (human-shaped forms) and the characterization dimension being the arousal level. The aggregation dimension we used were months, weeks, days of the week, hours of the day, subjects of calendar activities, and locations of calendar activities. Additionally, the users could also select their own views in LifelogExplorer.

For each of the views we first asked the user to explain what he can see in the view following a talk-aloud protocol. If anything was unclear, we provided an explanation. Next, we asked the participants to relate to the presented information by describing their perception for the specific aggregation presented in the view (in this procedure, we referred to the calendar if it was needed). To keep this phase focused, we picked a number of specific aggregations, that were particularly "red" or "green". In the next step, we asked if there is anything contradictory to the expectations. Finally, we asked if any of 
the information visualized could be used in practice, and how the person would use it.

Results: As a result of this evaluation we collected a number of comments regarding the views, the data and other aspects of the tool and the study procedure. Since the interview was semi-structured, the participants were allowed to comment on other topics, not covered by our questions. We illustrate the typical comments on the views generated for one of the participants (renaming is applied for privacy reasons). Fig. 4 shows a view on the user's data where the aggregation is performed on the days of the week, in principle, following the time line, but also making use of the cyclic nature of human life, revealing some weekly patterns (for the eight weeks of monitoring). An example of the view on the arousal related to calendar events aggregated by their subject with filtering applied is presented in Fig. 5. There one can see what people the user met and the arousal during these activities.

The subject-aggregated views were perceived as very useful: "This actually was really interesting. I actually learned. For instance, about the Club meetings. I really learned that the experience I have there is indeed reflected in the stress levels. It is an eye opener for me." "I learned which things on Monday night are stressful and I perhaps should relax and do something about that. That is very good to see." "I learned that teaching through video conferencing [DTA remote course] is really different from teaching in class [DTA lecture]."

The tool was also perceived as useful for reflection about the relations between people. "It is interesting to see these differences. It is like the one with Mark is completely red! Meetings with Frank are relaxed, but sometimes we are excited, because we have an interesting new idea, new functionality and there is higher excitement. Yes, that is excitement!" "The data that I see about the performance evaluations. The stress level in these job performance evaluations says much more about what the performance evaluation really was like. Much more than what is written on the paper. (...) It does not lie! (Laugh!) The report may lie, but the stress level does not lie."

One of the most important outcomes from the case study was the conclusion that the tool has potential for the use on the longer term, since the users want to see how the things in their lives develop: "I'd love to have more data, do this over a number of weeks and see whether this pattern reemerges."

The options provided in the tool give already a good coverage of the users interests in our case study:

Q: "What other views would you like to see?"

A: "I think these are pretty much the types of views that I would like to see. For activity, for day. For hours aggregated over all the days, I did not find that to be really relevant. Although it could be to some people. It shows when are you really getting up, when does your activity really start. For some people it could even mean like,... you think you work many hours a day, but look! (Laugh!). Given the level of stress, you really are only working hard for these many hours".

\section{B. Study with teachers of a vocational school}

In this study we collected data from five teachers for a period of seven weeks. The data collection was carefully monitored, but the participants were not supervised. We collected
33 days of measurements with 203 hours of measurements, per participant on average. As in the study at the university, we collected information about calendar activities (meeting, lessons, etc.), resulting in 165 calendar entries per person, on average. Additionally, the participants could indicate their emotional state per calendar entry using the SAM questionnaire, extended with the fatigue scale. They used this option for 88 calendar entries per participant, on average. Out of all the calendar entries indicated in the study period, on average 127 took place when a participant was also wearing the measurement device, which amounts to $76 \%$ of coverage of calendar activities by sensor measurements. At the end of the study, we processed all the collected data and aligned it. For one of the participants almost $70 \%$ of the sensor measurements have been lost due to artifacts in the signal and consequently we excluded this participant from the part of the evaluation concerned with selfreflection and self-coaching, providing the participant with the views based on the data available, explaining their limitations, and asking to comment on their usefulness under assumption that the data presented there would be complete enough.

Evaluation procedure: We performed the evaluation following a mixed protocol, with semi-structured interviews containing open questions for collecting qualitative feedback, and closed questions for gathering quantitative responses. The goal was to evaluate if the generated views are: 1) understandable, 2) reflect user's perception, 3) give new information, 4) trigger self-reflection and ideas for stress coping strategies. In total, 44 different views per person were generated, each depicting combinations of different dimensions in the data and filtering options. For each participant we picked 10 views on his/her data to be used in the evaluation. The views were presented starting from more general to more detailed views. For each view, we first collected comments following a talk-aloud protocol, without providing any explanations. After that we asked for comments concerning specific aggregations (circles) visualized. These were different per user, due to the personal nature of the data. In general, however, we selected the aggregations that stood out as being either very "red" or very "green". Finally, we asked each participant to rate the view by answering four questions on a 5-point Likert scale (from "Strongly disagree" to "Strongly agree"), and one question on a categorical Yes/No scale. At the end, after presenting all the views, we asked a number of closed questions, answered on the same 5-point Likert scale, to gather overall impression about the presented visualization.

Results: We give a brief summary of the evaluation results.

"I think that the presented views are useful for me": 3 participants responded "Agree", and 1 responded "Neutral". For the participants that agreed, they indicated that they would use it for "discussing with other people how to avoid stress" or to "improve time management". The participant that was neutral indicated that the views were interesting to see, but he did not learn anything new from them.

"I feel that the presented views gave me new information": 2 participants responded "Agree", and 2 responded "Neutral". We further asked for explanation what kind of new information they learned. Among the representative responses were: "I did not expect that [xx] work can be so stressful". An interesting conclusion was triggered by Fig. 6, showing the share of stressed activities to go through the day for one of 


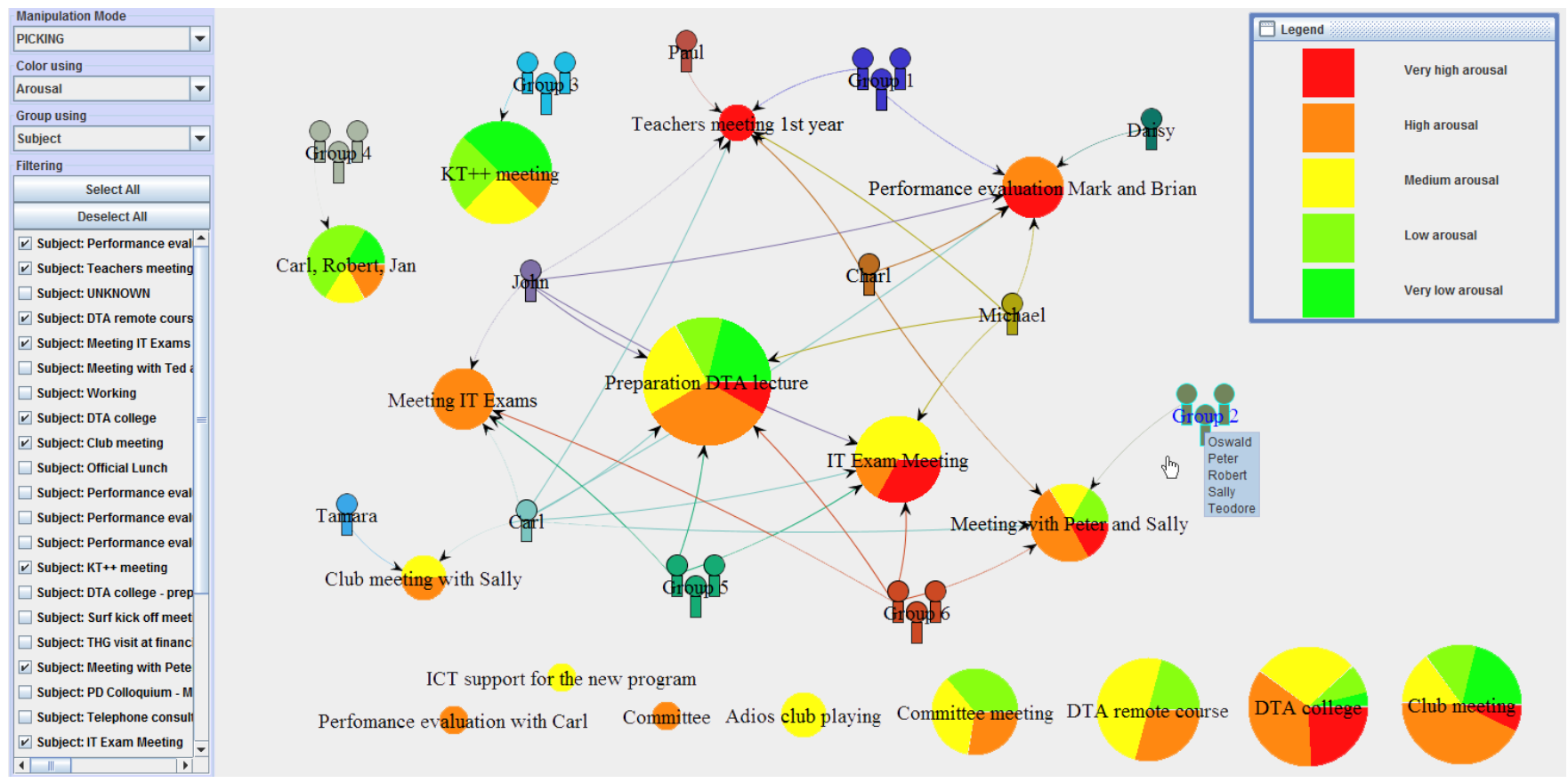

Fig. 5. A view with aggregation on subjects of calendar events with filtering applied.

the participants. He realized that it is his patterns indeed, and suggested an explanation for it: this participant turned out to work through the lunch breaks, which might effects his stress levels in the rest of the day indeed.

"I think I could apply what I have learned to improve some aspects of my life": 2 participants responded "Agree", and 2 responded "Disagree". Those who agreed indicated, the following self-coaching advices among others: "Maybe I should take lunch breaks", "Perhaps I should reconsider my involvement in the [xx] activity". Participants who did not feel they have learned anything that would allow them to improve any aspect of their lives, explained that they were mostly aware of their stress and its reasons and the views just confirmed what they already knew.

In addition to the summary responses related to participants perception, we also asked them to rate each view separately. We were able to see that the number of views that the participants indicated as useful to learn from varies greatly, with an average of $42.5 \%$ of the views indicated as useful to learn from. Following on that we found that "hours of the day" and "subjects of calendar activities" views were the two most useful views for most of our participants. For the statement "this view is useful for me", one participant answered "Strongly Agree" and 4 participants "agree" for the hours of the day view, while for the "Calendar activities" view 1 participant answered "Strongly Agree", three answered "Agree", and one was "Neutral".

In conclusion, the participants found the views informative, useful, and sometimes even revealing. Some participants, following their self-reflection, were able to generate self-coaching advices in order to better balance their workload. Curiously enough, most of the participants focused especially on the "red" areas, which represented more stressful periods, in most cases disregarding more "green" areas. Our current hypothesis

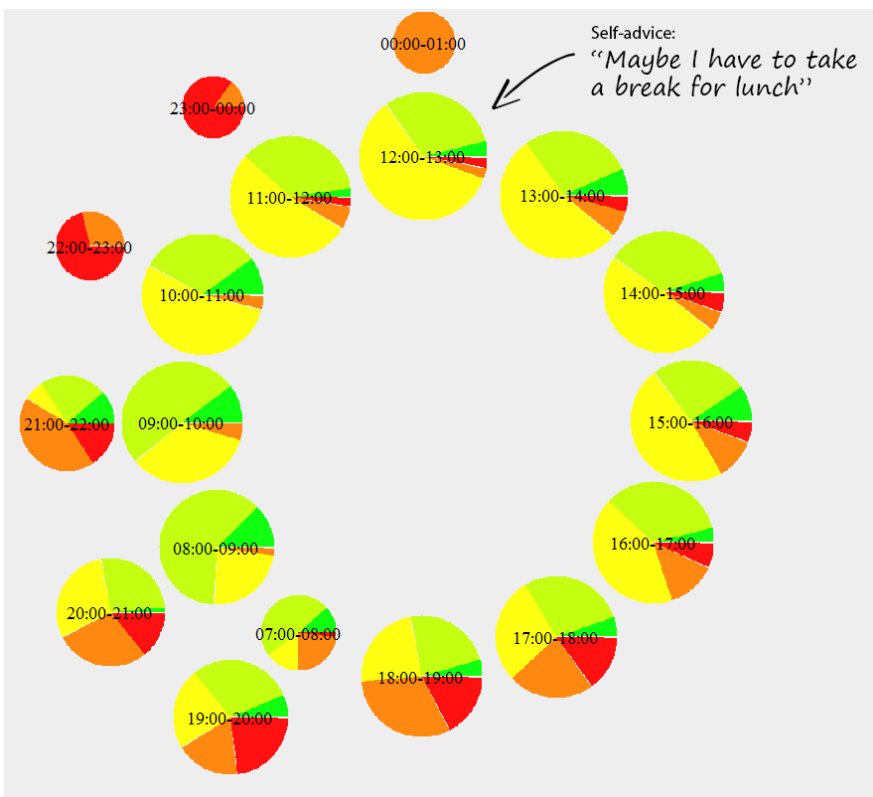

Fig. 6. A view on hours of the day from one of the school teachers, who indicated: "Maybe I have to take lunch breaks" in reaction to this view.

is that people pay more attention to the red color as indicating importance, danger or warning, and treat green as safe and correct, hence not worth attention. In terms of self-reflection, we consider the "green" areas as equally important, because they represent the relaxation moments for a person. We want to check how people will react to the views with a different color scale, e.g. ranging from light-blue to dark-blue, and whether it will result in drawing more attention to the areas of relaxation from which they could learn as well.

Additionally, we asked the users to compare some views on 
their data in LifelogExplorer with analogous views presented as stacked bar chart in terms of simplicity, informativeness, understandability and preference of use. We expected the LifelogExplorer to be perceived as a bit more complex, due to additional secondary dimension, but also to be more informative. Contrary to our expectations 4 out of 5 of our participants indicated the LifelogExplorer views as being simpler, and 3 out of 5 found them easier to understand. When asked for explanation, they indicated that it is easier to compare the stress level between different aggregations using circles than to relate heights of different parts of a stacked bar chart to each other. They also found the LifelogExplorer view to be especially clear in case there were many aggregations (circles) present, in which case the stacked bar chart becomes very "squeezed". The participant who preferred the stacked bar chart visualization indicated that it is easier to compare absolute time spent in each state using this view. For informativeness, 4 out of 5 participants considered LifelogExplorer views to be more informative, with one of the reasons being the presence of the secondary dimension that provided context, which some of the participants found very helpful in relating the stress level to a particular event in the past. Finally 4 out of 5 participants indicated they would prefer to use LifelogExplorer views over a stacked bar chart representation.

\section{CONClusions And Future WORK}

In this paper we presented our tool for facilitating understanding, reflecting on, and properly coping with stress. We make use of recent developments in sensor technologies that allow for unobtrusive, automated collection of information relevant for stress experienced in everyday life and show this information in relation to context information. We see the strength of our tool in the ability to visualize relations between different dimensions of the information space while preventing clutter, providing focus and using personally meaningful cues.

We applied our approach in two different practical contexts, focusing on stress experienced by busy academic staff members at a university, and by teachers of a vocational school. We learned that the views generated by LifelogExplorer are perceived as useful, informative, enable recollection and selfreflection in practical conditions. Finally we observed that the information presented in the views is, in many cases, actionable, and triggers self-coaching advices.

To further ease data exploration, we plan to improve interaction with our tool by adding a zoom in/zoom out functionality for easy switching between different abstraction levels. We also want to extend the tool with a possibility to watch the trends, by comparing "the same views" for several time periods. This functionality will enable the tracking of one's progress in a specific time period and facilitate monitoring the success of stress coping strategies applied.

\section{ACKNOWLEDGMENT}

The authors would like to thank EIT ICTLabs for financing the project, HumanCapitalCare for facilitating access to users, and staff members from TU/e and teachers from ROC Eindhoven for participating in our studies.

\section{REFERENCES}

[1] A. Stanley and P. Clipsham, "Information overload-myth or reality?" in IT Strategies for Information Overload (Digest No: 1997/340), IEE Colloquium on. IET, 1997, pp. 1-4.

[2] T. Cox, Stress research and stress management: Putting theory to work. HSE Books Sudbury, 1993.

[3] S. Praissman, "Mindfulness-based stress reduction: A literature review and clinicians guide," Journal of the American Academy of Nurse Practitioners, vol. 20, no. 4, pp. 212-216, 2008.

[4] M. Engin, A. Demirel, E. Engin, and M. Fedakar, "Recent developments and trends in biomedical sensors," Measurement, vol. 37, no. 2, pp. 173-188, 2005.

[5] J. Bakker, L. Holenderski, R. Kocielnik, M. Pechenizkiy, and N. Sidorova, "Stess@ work: From measuring stress to its understanding, prediction and handling with personalized coaching," in Proceedings of the 2nd ACM SIGHIT International health informatics symposium. ACM, 2012, pp. 673-678.

[6] P. Paoli, D. Merllié, and F. per a la Millora, Third European survey on working conditions 2000. European Foundation for the Improvement of Living and Working Conditions, 2001.

[7] K. Glanz and M. Schwartz, "Stress, coping, and health behavior," Health behavior and health education: theory, research, and practice. 4th edition. San Francisco (CA): John Wiley and Sons, Inc, pp. 211-236, 2008.

[8] N. Sharma and T. Gedeon, "Objective measures, sensors and computational techniques for stress recognition and classification: A survey," Computer Methods and Programs in Biomedicine, 2012.

[9] J. Westerink, M. Ouwerkerk, G. de Vries, S. de Waele, J. van den Eerenbeemd, and M. van Boven, "Emotion measurement platform for daily life situations," in Affective Computing and Intelligent Interaction and Workshops, 2009. ACII 2009. 3rd International Conference on. IEEE, 2009, pp. 1-6.

[10] E. Freeman and S. Fertig, "Lifestreams: Organizing your electronic life," in AAAI Fall Symposium: AI Applications in Knowledge Navigation and Retrieval, 1995, pp. 38-44.

[11] J. Gemmell, G. Bell, and R. Lueder, "Mylifebits: a personal database for everything," Communications of the ACM, vol. 49, no. 1, pp. 88-95, 2006.

[12] E. Singer, "The measured life," Technology Review, vol. 114, no. 4, pp. $38-45,2011$

[13] I. Li, A. Dey, and J. Forlizzi, "A stage-based model of personal informatics systems," in Proceedings of the 28th international conference on Human factors in computing systems. ACM, 2010, pp. 557-566.

[14] D. McDuff, A. Karlson, A. Kapoor, A. Roseway, and M. Czerwinski, "AffectAura: an intelligent system for emotional memory," in Proceedings of the 2012 ACM annual conference on Human Factors in Computing Systems. ACM, 2012, pp. 849-858.

[15] P. Sanches, K. Höök, E. Vaara, C. Weymann, M. Bylund, P. Ferreira, N. Peira, and M. Sjölinder, "Mind the body!: designing a mobile stress management application encouraging personal reflection," in Proceedings of the 8th ACM conference on designing interactive systems. ACM, 2010, pp. 47-56.

[16] Y. Ayzenberg, J. Hernandez Rivera, and R. Picard, "Feel: frequent EDA and event logging-a mobile social interaction stress monitoring system," in Proceedings of the 2012 ACM annual conference extended abstracts on Human Factors in Computing Systems Extended Abstracts. ACM, 2012, pp. 2357-2362.

[17] U. Maurer, A. Smailagic, D. Siewiorek, and M. Deisher, "Activity recognition and monitoring using multiple sensors on different body positions," in Wearable and Implantable Body Sensor Networks, 2006. BSN 2006. International Workshop on. IEEE, 2006, pp. 116-121.

[18] L. Bao and S. Intille, "Activity recognition from user-annotated acceleration data," Pervasive Computing, pp. 1-17, 2004.

[19] W. Boucsein, Electrodermal activity. Springer, 2011.

[20] R. Kocielnik, N. Sidorova, F. Maggi, J. Westerink, and M. Ouwerkerk, "Smart technologies for long-term stress monitoring at work," in Proceedings of the 26th IEEE international Symposium on Computer-Based Medical Systems. CBMS. IEEE, 2013. 